\title{
Use of regional clinical data to identify veterans for a multi-center osteoporosis electronic consult quality improvement intervention
}

\author{
Cathleen Colón-Emeric ${ }^{1}$, Richard Lee $^{1}$, Karen Barnard $^{1}$, Megan Pearson $^{2}$, Kenneth W. Lyles ${ }^{1}$ \\ 1. Durham VA Geriatric Research Education and Clinical Center/ Duke University Health System, Durham, NC, USA. 2. \\ Durham VA Geriatric Research Education and Clinical Center, Durham, NC, USA
}

Correspondence: Cathleen Colón-Emeric. Address: Durham VA Geriatric Research Education and Clinical Center/ Duke University Health System, 508 Fulton St., GRECC 182, Durham, NC 27705, USA. E-mail: Colon001@mc.duke.edu

Received: August 28, 2012

Accepted: October 9, 2012

Online Published: November 5, 2012

DOI : $10.5430 /$ jha.v2n1p8

URL: http://dx.doi.org/10.5430/jha.v2n1p8

\section{Abstract}

Background: Electronic medical record systems can rapidly identify fracture patients so that healthcare systems can target osteoporosis treatment programs. However, it is not clear what proportion of such patients are actually eligible for treatment.

Method: In 3 Veterans Affairs Medical Centers, a secondary fracture prevention electronic screening protocol was developed and preceded in 3 stages. First, all patients with a fracture-related ICD-9 or CPT code for fracture over the preceding 6 months were identified using a structured query language (SQL) server report run regularly on regional clinical data. Additional data was obtained automatically at this stage, and patients were excluded if they were already on bisphosphonate, their fracture was facial or digital, they did not have a primary care provider, they were under age 50 years, or had died.

In a second stage, chart abstraction was completed by the project director. Patients were excluded if their fracture occurred after high-impact trauma, the coded fracture was not confirmed on radiograph, the fracture occurred more than 10 years previously, bone density screening had already been obtained, the fracture was pathologic, the patient was receiving palliative care, or the patient had been offered and declined therapy.

In the final stage, remaining patients were referred to a bone specialist who reviewed the medical record and generated an electronic consult to the primary provider that gave recommendations for further evaluation and management consistent with current guidelines.

Results: Among 986 screened Veterans with ICD9 fracture code within the study period, 841 (85\%) were ultimately excluded from further intervention. A majority $(\mathrm{n}=574,68 \%)$ were excluded in the first, automated screening stage [no primary provider (22\%), age under 50 years (38\%), already on a bisphosphonate (12\%), fracture facial or digital (25\%), patient had died (3\%)]. Chart abstraction was required to exclude 267 (32\%) prior to physician review [high trauma (37\%), remote injury or no evidence of fracture (36\%), palliative care (9\%), and other reasons (18\%)]. One hundred three consults were completed, with 80 (78\%) recommending osteoporosis treatment or bone mineral density (BMD) testing.

Conclusion: An electronic screening tool was effective at a regional level in identifying recent fracture patients for secondary osteoporosis intervention, but many (85\%) are ultimately not eligible for additional interventions. Most exclusion (68\%) can be made without additional chart abstraction.

\section{Key words}

Electronic medical record, Osteoporosis, Health services research 


\section{Background}

The occurrence of a low trauma fracture identifies patients who are at very high risk for additional fractures and increased healthcare costs over the next 5 years ${ }^{[1,2]}$. Treatment with bisphosphonates, calcium, and vitamin $\mathrm{D}$ after low trauma fractures reduces subsequent fracture rates, and in the case of hip fractures, mortality ${ }^{[3,4]}$. However, low rates of osteoporosis evaluation and treatment after fracture have been documented in multiple settings and countries, especially among men ${ }^{[5-8]}$. In 2010, the Office of the Inspector General reviewed osteoporosis care among Veterans with low trauma fracture, and found that only $24 \%$ received appropriate care. System-wide quality improvement interventions were advocated ${ }^{[9]}$.

Several types of quality improvement interventions to improve osteoporosis treatment after a fracture have been tested. Education/reminder interventions, where patients and/or primary care providers receive mailed or faxed information about secondary fracture prevention following a low trauma fracture, appear to have limited or no impact on testing and treatment rates ${ }^{[10-14]}$. Providing osteoporosis testing and treatment services within orthopedic clinics appears to be quite effective in several randomized trials and a meta-analysis ${ }^{[15-18]}$, but requires substantial resources and is not widely available. Finally, utilizing a nurse care manager who is responsible for identifying and arranging evaluation and treatment of all fracture patients within a hospital system appears to substantially improve osteoporosis care at modest cost ${ }^{\text {[19-21]; }}$; however, it may be inefficient for small medical centers with lower fracture volume to provide such services. Moreover, osteoporosis testing and treatment decisions may be more complex in men or other patients with multiple co-morbidities, requiring physician input. Centralization of fracture coordinator services, with an opportunity for physician review when needed, may therefore be the optimal strategy in healthcare systems where there are multiple centers with variable fracture volumes, and complex patient characteristics.

The purpose of this article is to describe the use of a regional clinical data repository to identify patients with recent fracture for inclusion in a multi-center osteoporosis e-consult intervention. We describe the screening process in terms of the logistics of obtaining regional fracture reports, the yield of eligible patients at each screening step, and reasons for exclusion. With health care centers adopting centralized access to electronic clinical records, this process may be relevant to other clinical diagnoses.

\section{Methods}

\subsection{Patient eligibility}

Patients receiving an osteoporosis e-consult were over age 50 years, had sustained a low trauma fracture within the last 6 months (fall from standing height or less), and had a primary care provider within the VA system. Exclusions included fractures not considered osteoporotic (facial, skull, digital, or pathologic fracture), an active prescription for a bisphosphonate, or estimated life expectancy 1 year or less (hospice care, metastatic cancer, end-stage heart or lung disease).

\subsection{Patient identification - data warehouse report tool}

A SQL Server report was developed to identify fracture-related International Classification of Disease (ICD9) and Current Procedural Terminology (CPT) codes entered over a 6 month period (CPT codes: 27230, 27232, 27235, 27236,27238, 27240, 27244, 27245, 27246, 27248; ICD9 codes 733.93 through 733.95; 767.3; 800 through 829; V54.13). Additional data pulled for the report included age, sex, assigned primary care provider, prescription for calcium and vitamin D supplements, prescription for bisphosphonate, and mortality status. The SQL server report is run every other week on 
regional data (data warehouse, VISN6, clinical data uploaded with 1 week time delay), and can be downloaded onto an excel spreadsheet by project staff.

\subsection{Patient screening}

The screening process is outlined in Figure 1. Screening proceeded in 2 stages. First, using the SQL server report, patients were excluded if they were already on bisphosphonate, if they were under age 50 years, had died, or if their fracture was non-osteoporotic (facial, digital, skull). Patients who did not have an assigned VA primary care provider were also excluded; some Veterans choose to use a primary care provider outside the VA system, but these community providers do not use the VA electronic medical record, nor is it possible to identify which community provider is caring for the Veteran.

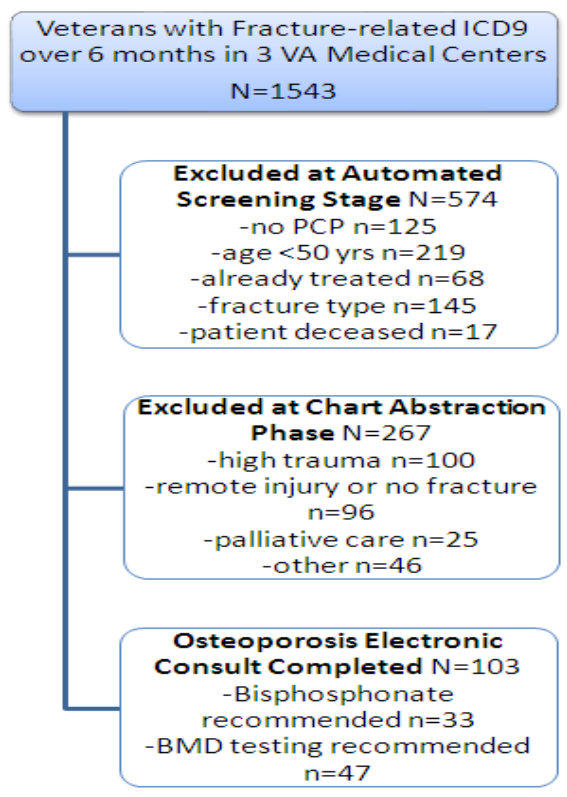

Figure 1. Flow diagram of regional patient identification, screening, and consultation process

In a second stage, electronic medical record screening was completed remotely by the project director. Patients were excluded if their fracture occurred after high-impact trauma, the coded fracture was not confirmed on radiograph, the fracture occurred more than 10 years previously, bone mineral density screening had already been obtained, the fracture was pathologic, the patient was receiving palliative care, or the patient had been offered and declined therapy. Remaining patients were referred for an osteoporosis e-consult.

\subsection{E-consult process}

A physician bone specialist (endocrinologist or geriatrician) reviewed the electronic medical record and generated a consult note. Specifically, the physician reviewed pertinent laboratory data (creatinine clearance, serum calcium level, 25(OH) Vitamin D level, testosterone, complete blood counts), other clinical risk factors for fracture (low BMI, corticosteroid use, falls, co-morbidities associated with fractures), and prior osteoporosis treatment if any. The physician then used an electronic note template to summarize the relevant data, and provide recommendations for initiation of osteoporosis treatment and/or further evaluation. Recommendations were based on current clinical practice guidelines from the National Osteoporosis Foundation and the VA ${ }^{[22,23]}$.

The note was flagged to be electronically co-signed by the patient's primary care provider. Concurrently, an encrypted email notifying the provider that their patient had a recent fracture and that an e-consult needed their attention was sent. If the note was not co-signed within 1 month, a follow-up email reminder was sent. 


\subsection{Data collection and follow-up}

The project director tracks all e-consults 3 and 6 months after completion to determine whether the patient has a new order for bisphosphonate, other osteoporosis pharmacotherapy, calcium supplement, vitamin D supplement, or BMD testing. Six-month bisphosphonate treatment rates after fracture at the medical center level before and after e-consult program initiation will be measured, and also compared to 3 similar centers not receiving the program. Comparison medical centers were selected on the basis of size, geographic proximity to intervention proximity, and academic affiliation status. Because individual patient-level variables available for this clinical demonstration project are limited, no adjustment for case-mix will be completed. Instead, we will use a pre-post design comparing within-center changes over time.

Per VA national policy, this program was considered non-research and IRB approval was not required. Permission for publication of results was obtained from the Medical Center Director.

\section{Results}

In the 3 participating VA Medical Centers, 1543 unique individuals had a fracture-related ICD9 or CPT code in the 6 months between 3/1/11 and 8/31/11, and 986 veterans have been screened for the program to date, with 557 not yet screened. Of the screened patients, 841 (85\%) were ultimately excluded from further intervention. A majority $(n=574$, $68 \%$ of the excluded patients) were excluded in the first, automated screening stage with SQL server data [no primary provider (22\%), age under 50 years (38\%), already on a bisphosphonate (12\%), fracture facial, digital, or skull (25\%), patient had died (3\%)].

Table 1. Baseline Characteristics of Veterans Receiving Electronic Consult

\begin{tabular}{ll}
\hline *Characteristics & $\mathbf{N}=\mathbf{9 9}$ \\
\hline Age, mean (SD), range & $71.2(12.33) 51-96$ \\
Under 70, No.\% & $53(53.5)$ \\
Race, Caucasian, No.\% & $69(69.7)$ \\
Race, African American, No.\% & $26(26.3)$ \\
Race, Unknown, No.\% & $4(4.0)$ \\
Male sex, No.\% & $87(87.9)$ \\
Female sex, No.\% & $12(12.1)$ \\
$* *$ BMI, mean (SD), range & $26.44(5.91) 14.94-42.96$ \\
Number of prior Dexa, mean (SD) & $13(13.1)$ \\
$* *$ Recent eGFR, mean (SD), range & $61.8(27.1) 9.5-178.5$ \\
$* *$ Recent serum Calcium, mean (SD), range & $9.0(0.5) 7.9-10.0$ \\
$* * *$ Recent serum Calcium, mean (SD), range & $27.6(12.48) 4.6-73.1$ \\
\hline
\end{tabular}

*Two patients age 40 excluded.

**Two values not available.

***Forty-four not available.

Chart abstraction was required to exclude 267 (32\% of the excluded patients) prior to physician review [high trauma (37\%), remote injury or no evidence of fracture (36\%), palliative care (9\%), and other reasons (18\%)]. The abstraction was completed by a trained, non-clinical project director, and took on average of 10 minutes per patient (range 5-12 minutes).

One hundred forty-five veterans have been referred for physician e-consults, and 103 have been completed as of May 1, 2012. The e-consults required an average of 12 minutes to complete (range 10-20). Approximately one-third (32\%) recommended initiation of bisphosphonate, and half (46\%recommended BMD testing. Other frequent recommendations include calcium and vitamin D supplements, consideration of bisphosphonate therapy after re-testing of creatinine clearance in patients with borderline renal function, or referral to metabolic bone clinic for those requiring additional 
testing. As of May 1, 2012, 50\% of the recommendations for bisphosphonate prescriptions and $40 \%$ of the recommendations for BMD testing have been ordered by the primary care providers. Comparison of the facility-level treatment rates with pre-program rates and with concurrent rates in control facilities will occur 6 months after full program implementation, and will be reported in a separate manuscript).

\section{Discussion}

We have demonstrated the feasibility of a regional, multi-center quality improvement program to improve osteoporosis treatment after low-trauma fracture. The centralized, remote coordination of low-frequency but highly cost-effective services such as secondary fracture prevention may be an effective way to improve care within large health care systems while optimizing resource utilization. Prior studies of the osteoporosis care-coordinator model in Canada have estimated that the intervention costs approximately $\$ 56$ per patient; for every 100 patients who are case managed, 6 fractures (including 4 hip fractures) are prevented, 4 quality-adjusted life-years are gained, and $\$ 260,000$ is saved by the health care system $^{[13]}$. While these results need to be replicated in the U.S., a similar program is likely to be economically attractive or cost-savings given the low cost and effectiveness of secondary fracture prevention therapies and the high cost and morbidity of fractures.

These centralized, remote clinical services require the leverage of available health information technology for success. Use of regional or even national clinical data warehouses can allow for rapid identification of the target population, as well as a first-level screening for exclusion criteria. Remote review of pertinent clinical data by experts can allow for clinical decision making without the need for an in-person visit with the patient in many situations. Rapid, bi-directional communication with primary care providers can occur through secure email or messaging systems, and recommendations and actions can be documented in an e-consult note. Monitoring of program outcomes can occur to a large extent using available electronic data.

Current limitations to the use of health information technology for multi-center quality improvement interventions should also be considered. The patient identification process depends on accurate and consistent coding by clinicians. Although fracture coding has been documented to be more than $90 \%$ accurate in a Medicare population ${ }^{[24]}$, it may be less so in the VA setting or in other chronic conditions. Some important outcomes that clinicians may wish to identify are rarely coded and would require natural language processing (NLP) of text fields in order to identify potential cases. For example, 2/3 of vertebral fractures are clinically silent, but are frequently observed incidentally on chest radiographs. While it is technically possible to screen radiograph test results for vertebral fracture using NLP, the programs take a great deal of time to run and are thought to incur a risk of slowing or disrupting normal system operations. Other clinical data that might be readily used for screening regional databases include laboratory results and medication prescriptions.

Once potential patients are identified, logistical barriers designed to protect health information may hamper the quality improvement team's ability to access data remotely and communicate effectively with providers. In our program, permissions from multiple offices or committees in each medical center had to be secured in order for our team members to access clinical data and write e-consult notes. These included the medical center director, the information security officer, the credentialing committee, the medical records committee, the tele-health coordinator, and health information services, with different requirements in each center. Because the remote clinicians were not fully credentialed by each medical center, only limited access to the electronic record was granted, requiring the construction of a "health summary tab" by the local health information services to access the required data fields. If centralized care coordination quality improvement programs such as ours are found to be effective and adopted widely by the VA, streamlined and consistent processes to provide remote access to health information technology would be critically important.

This program has been in operation for 6 months; its impact on osteoporosis quality indicators and formal provider satisfaction surveys will be completed within the next year. Ideally, larger randomized trials of this QI model will be 
needed to establish its effectiveness and impact on fracture rates and cost. However, we have established the feasibility and acceptability of using regional clinical data to identify patients for a remote, electronic consultation program. This centralized case-management quality improvement model may be useful in other low frequency but high cost conditions such as HIV, Hepatitis C, stage 4-5 Chronic Kidney Disease, or others.

\section{Acknowledgements}

This project was funded by the VISN 6 Specialty Care Office. We are grateful for the assistance of Dr. Kim Voss, Dr. Parag Dalsania, Dr. Peter Chung, and Ms. Katherine McMullin.

\section{Conflicts of interests}

This project was funded by the VA VISN-6 Office of Specialty Care.

The authors report the following potential conflicts of interest:

-Drs. Colón-Emeric, O’Connor and Lyles are co-owners of Biscardia, LLC and co-inventors of US Provisional Patent Application: "Bisphosphonates and Mortality Reduction" and a second US Provisional Patent relating to bisphosphonates and cardiovascular disease, "Bisphosphonate Compositions and Methods for Treating Heart Failure".

-Dr. Colón-Emeric is an advisory board member of Amgen, and a consultant for Novartis on osteoporosis-related topics.

-Dr. Lyles is an advisory board member for Amgen, a consultant and speaker for Amgen and Novartis, Co-Inventor of US Patent Application: "Methods for preventing or reducing secondary fractures after hip fracture”, Number 20050272707, and Inventor of US Patent Application: "Medication Kits and Formulations for Preventing, Treating or Reducing Secondary Fractures After Previous Fracture” Number 12532285.

\section{References}

[1] Colon-Emeric C, Kuchibhatla M, Pieper C, Hawkes W, Fredman L, Magaziner J, et al. The contribution of hip fracture to risk of subsequent fractures: data from two longitudinal studies. Osteoporos Int. 2003; 14(11): 879-83. PMid:14530910 http://dx.doi.org/10.1007/s00198-003-1460-x

[2] Zimmerman S, Chandler J, Hawkes W, Sloane P, Hebel J, Magaziner J, et al. Effect of fracture on the health care use of nursing home residents. Arch Int Med. 2002; 162(13): 1502-8. http://dx.doi.org/10.1001/archinte.162.13.1502

[3] Lyles K, Colón-Emeric C, Magaziner J, Adachi J, Pieper C, Mautalen C, et al. Zoledronic acid and clinical fracture and mortality after hip fracture. New Engl J Med. 2007; 357: 1799-809. PMid:17878149 http://dx.doi.org/10.1056/NEJMoa074941

[4] Beaupre L, Morrish D, Hanley D, Maksymowych W, Bell N, Juby A, et al. Oral bisphosphonates are associated with reduced mortality after hip fracture. Osteoporosis International. 2011; 22(3): 983-91. PMid:21052642 http://dx.doi.org/10.1007/s00198-010-1411-2

[5] Feldstein AC, Nichols G, Orwoll E, Elmer PJ, Smith DH, Herson M, et al. The near absence of osteoporosis treatment in older men with fractures. Osteoporosis International. 2005; 16(8): 953-62. PMid:15928798 http://dx.doi.org/10.1007/s00198-005-1950-0

[6] Fraser LA, Ioannidis G, Adachi JD, Pickard L, Kaiser SM, Prior J, et al. Fragility fractures and the osteoporosis care gap in women: the Canadian Multicentre Osteoporosis Study. Osteoporosis International. 2011; 22(3): 789-96. PMid:20683706 http://dx.doi.org/10.1007/s00198-010-1359-2

[7] Gunter MJ, Beaton SJ, Brenneman SK, Chen Y-T, Abbott TA, 3rd, Gleeson JM. Management of osteoporosis in women aged 50 and older with osteoporosis-related fractures in a managed care population. Disease Management. 2003; 6(2): 83-91. PMid:14577902 http://dx.doi.org/10.1089/109350703321908469

[8] Petrella RJ, Jones TJ. Do patients receive recommended treatment of osteoporosis following hip fracture in primary care? BMC Family Practice. 2006; 7: 31. PMid:16684358 http://dx.doi.org/10.1186/1471-2296-7-31

[9] Management of Osteoporosis in Veterans with Fractures. Report of the Office of the Inspector General. Washington, D.C. 2010; $1-15$.

[10] Bliuc D, Eisman JA, Center JR. A randomized study of two different information-based interventions on the management of osteoporosis in minimal and moderate trauma fractures. Osteoporosis International. 2006; 17(9): 1309-17. PMid:16804739 http://dx.doi.org/10.1007/s00198-006-0078-1 
[11] Cranney A, Lam M, Ruhland L, Brison R, Godwin M, Harrison MM, et al. A multifaceted intervention to improve treatment of osteoporosis in postmenopausal women with wrist fractures: a cluster randomized trial. Osteoporosis International. 2008; 19(12): 1733-40. PMid:18629567 http://dx.doi.org/10.1007/s00198-008-0669-0

[12] Jaglal SB, Hawker G, Bansod V, Salbach NM, Zwarenstein M, Carroll J, et al. A demonstration project of a multi-component educational intervention to improve integrated post-fracture osteoporosis care in five rural communities in Ontario, Canada. Osteoporosis International. 2009; 20(2): 265-74. PMid:18521649 http://dx.doi.org/10.1007/s00198-008-0654-7

[13] Majumdar SR, Johnson JA, Lier DA, Russell AS, Hanley DA, Blitz S, et al. Persistence, reproducibility, and cost-effectiveness of an intervention to improve the quality of osteoporosis care after a fracture of the wrist: results of a controlled trial. Osteoporosis International. 2007; 18(3): 261-70. PMid:17086470 http://dx.doi.org/10.1007/s00198-006-0248-1

[14] Colon-Emeric CS, Lyles KW, House P, Levine DA, Schenck AP, Allison J, et al. Randomized trial to improve fracture prevention in nursing home residents. American Journal of Medicine. 2007; 120(10): 886-92. PMid:17904460 http://dx.doi.org/10.1016/j.amjmed.2007.04.020

[15] Bogoch ER, Elliot-Gibson V, Beaton DE, Jamal SA, Josse RG, Murray TM. Effective initiation of osteoporosis diagnosis and treatment for patients with a fragility fracture in an orthopaedic environment. Journal of Bone \& Joint Surgery - American Volume. 2006; 88(1): 25-34. PMid:16391246 http://dx.doi.org/10.2106/JBJS.E.00198

[16] Kuo I, Ong C, Simmons L, Bliuc D, Eisman J, Center J. Successful direct intervention for osteoporosis in patients with minimal trauma fractures. Osteoporosis International. 2007; 18(12): 1633-9. PMid:17603741 http://dx.doi.org/10.1007/s00198-007-0418-9

[17] Sale JEM, Beaton D, Posen J, Elliot-Gibson V, Bogoch E. Systematic review on interventions to improve osteoporosis investigation and treatment in fragility fracture patients. Osteoporosis International. 2011; 22(7): 2067-82. PMid:21607808 http://dx.doi.org/10.1007/s00198-011-1544-y

[18] Johnson SL, Petkov VI, Williams MI, Via PS, Adler RA. Improving osteoporosis management in patients with fractures. Osteoporosis International. 2005; 16(9): 1079-85. PMid:15586267 http://dx.doi.org/10.1007/s00198-004-1814-z

[19] Majumdar SR, Johnson JA, Bellerose D, McAlister FA, Russell AS, Hanley DA, et al. Nurse case-manager vs multifaceted intervention to improve quality of osteoporosis care after wrist fracture: randomized controlled pilot study. Osteoporosis International. 2011; 22(1): 223-30. PMid:20358359 http://dx.doi.org/10.1007/s00198-010-1212-7

[20] Ward SE, Laughren JJ, Escott BG, Elliot-Gibson V, Bogoch ER, Beaton DE. A program with a dedicated coordinator improved chart documentation of osteoporosis after fragility fracture. Osteoporosis International. 2007; 18(8): 1127-36. PMid:17333450 http://dx.doi.org/10.1007/s00198-007-0341-0

[21] Sander B, Elliot-Gibson V, Beaton DE, Bogoch ER, Maetzel A. A coordinator program in post-fracture osteoporosis management improves outcomes and saves costs. Journal of Bone \& Joint Surgery - American Volume. 2008; 90(6): 1197-205. PMid:18519311 http://dx.doi.org/10.2106/JBJS.G.00980

[22] Dawson-Hughes B, Committee obotNOFG. A Revised Clinician's Guide to the Prevention and Treatment of Osteoporosis. Journal of Clinical Endocrinology \& Metabolism. 2008; 93(7): 2463-5. PMid:18544615 http://dx.doi.org/10.1210/jc.2008-0926

[23] Petzel R. Under Secretary for Health's Information Letter: Osteoporosis in Men. In: Affairs DoV, ed. Washington, DC: Veterans Health Administration. 2011.

[24] Campbell SE, Campbell MK, Grimshaw JM, Walker AE. A systematic review of discharge coding accuracy. Journal of Public Health. 2001; 23(3): 205-11. http://dx.doi.org/10.1093/pubmed/23.3.205 